\title{
A design of toxic gas detecting security robot car based on wireless path-patrol
}

\author{
Ho-Chih Cheng ${ }^{1}$, Min-Chie Chiu ${ }^{1, *}$, Kun-Fu Zeng ${ }^{1}$, and Che-Min $\mathrm{Chiu}^{2}$ \\ ${ }^{1}$ Department of Mechanical and Automation Engineering, Chung Chou University of Science and Technology, 6, Lane 2, Sec.3, \\ Shanchiao Rd., Yuanlin, Changhua 51003, Taiwan, R.O.C. \\ ${ }^{2}$ Institute of Biomedical Engineering, National Tsing Hua University, 101, Sec. 2, Kuang-Fu Rd., Hsinchu 30013, Taiwan, R.O.C.
}

\begin{abstract}
Because a toxic gas detecting/monitoring system in a chemical plant is not movable, a gas detecting/monitoring system will be passive and the detecting range will also be constrained. This invention is an active multi-functional wireless patrol car that can substitute for humans that inspect a plant' $s$ security. In addition, to widen the monitoring vision within the environment, two motors used to rotate a wireless IPCAM with two axes are presented. Also, to control the robot car's movement, two axis motors used to drive the wheel of the robot car are also installed. Additionally, a toxic gas detector is linked to the microcontroller of the patrol car. The detected concentration of the gas will be fed back to the server pc. To enhance the robot car's patrolling duration, a movable electrical power unit in conjunction with a wireless module is also used. Consequently, this paper introduces a wireless path-patrol and toxic gas detecting security robot car that can assure a plant's security and protect workers when toxic gases are emitted.
\end{abstract}

\section{Introduction}

Toxic gases are very often released in chemical plants. Most of the dangerous toxic gases are colorless, odorless, and tasteless. Some of them (methane (CH4), nitrogen dioxide (NO2), and hydrogen sulphide) emitted from the underground region $(\mathrm{H} 2 \mathrm{~S})$ are not easily detected by human senses and are flammable and lighter than air [1]. As investigated by Steenland et al.[2] and Lind [3], the complex nature of chemical plants increases the risks for maintenance workers who are often close to a broad variety of hazardous chemicals. In addition, fine particulate air pollution is harmful to humans $[4,5,6]$. Therefore, in order to ensure a worker's health, detecting toxic gases and fine particles is essential. In pass decades, traditional gas detecting/monitoring stations in chemical plants have also been established [7, 8]. But, because of their fixed location, they are passive and their detecting range is constrained. Additionally, to recognize gas categories, large gas monitoring systems, which include many gas detecting stations, have been presented [9]. However, the cost is huge. In order to overcome the above drawbacks, a robot car used to patrol and detect toxic gas emissions in a plant is presented.

The application of robots used in smart homes has been widely developed. In previous studies, Chiu et al. developed wireless vacuum cleaning systems by using a robot car [10]. In order to advance cleaning efficiency, the path-planning for a robot car using various artificial intelligent algorithms has been explored [11, 12, 13]. Also, to improve the electrical duration of the robot car, a wall plug-in power supply system [14] and a cable spooling system [15] have been established. To verify the ground purity of the vacuum cleaning work, groundpurity inspection, vision guidance, and positioning and path planning for a robotic cleaner has been explored [16, 17, 18]. Moreover, Chiu et al. has also applied the robot car as an auto-tracking carrier to an AGV using a wavevaried detecting method [19]. To ensure security at home, a real-time editing task service-oriented security robot has also been developed [20].

Here, in order to patrol and to detect toxic fumes within plants, a wireless path-patrol and toxic gas detecting security robot car is presented.

\section{Robot car system}

As indicated in Figure 1, the robot car system includes a PC control port, a stick control port, a visual port, a gas sensor, and a robot car. A toxic gas sensor (methane) connected to the microcontroller which is installed onto the robot car can detect the toxic gas and send back the data to server PC port via the blue tooth prorocol. In order to remotely monitor/control the robot car, a wireless USB AP router wirelessly connected to the IPCAM is adopted and connected to the server PC via the Wi-Fi protocol. In addition, a PS2 stick (stick control port) used to control the direction of the carrier (robot car) and the rotating angle of the servo motor (for the IPCAM) is connected to the server PC. The robot car is also wirelessly connected to the server PC via the blue tooth protocol. As indicated in Figure 2, in order to increase electrical duration during a patrolling mission, a power bank, a 5 Volt Ni-MH battery, is also installed onto the robot car.

\footnotetext{
Corresponding author: minchie.chiu@msa.hinet.net
} 


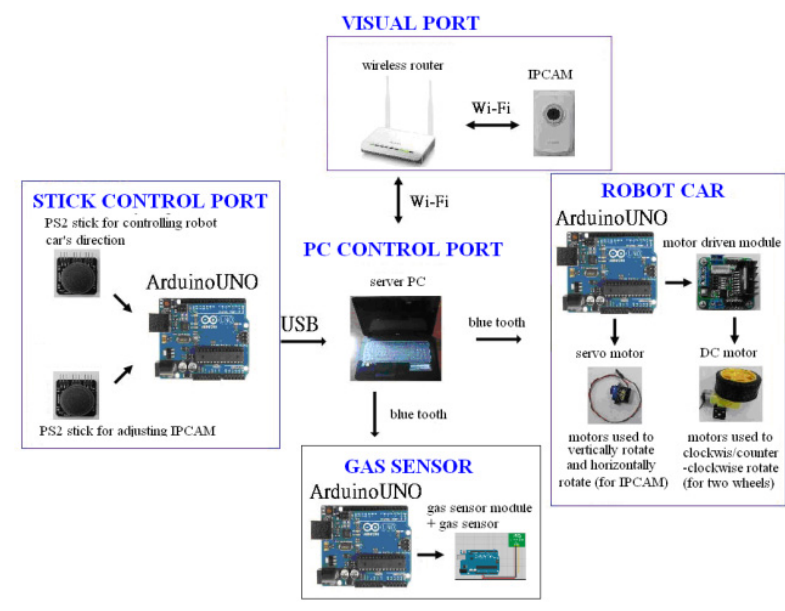

Fig. 1. Structure of the wireless path-patrol and toxic gas detecting security robot car.

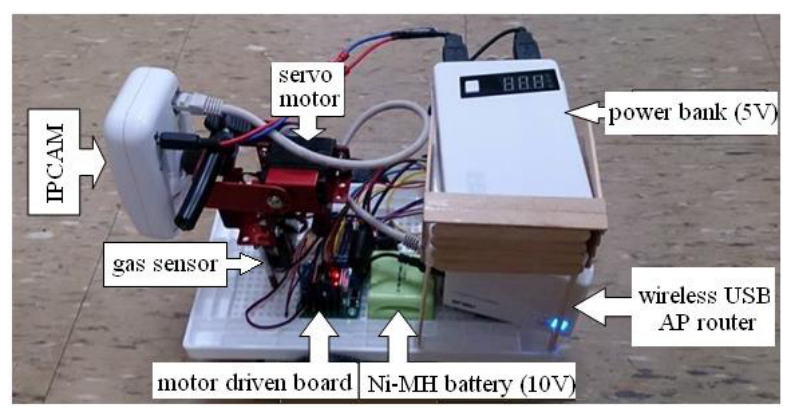

Fig. 2. Prototype of the wireless path-patrol and toxic gas detecting security robot car.

As shown in Figures 3 and 4, ArduinoUNO and Arduino sensor shield v5 single chips are adopted as the microcontroller. In addition, the PS2 stick module's functional diagram is depicted in Figure 5. The related functions of the PS2 stick is shown in Figure 6 . Hardware for the motor driven board and the Arduino expanded board is illustrated in Figure 7. Moreover, the electrical circuit for the blue tooth protocol is shown in Figure 8. For the pin connection, the corresponding pins of blue tooth with respect to Arduino expanded board is shown in Table 1. The corresponding pins of the motor driven board with respect to the Arduino expanded board is depicted in Table 2. Also, the corresponding pins of the PS2 stick board (for the carrier's motor control) with respect to the Arduino expanded board are illustrated in Table 3. Consequently, the corresponding pins of the PS2 stick board (for the IPCAM's servo motor control) with respect to the Arduino expanded board are listed in Table 4.

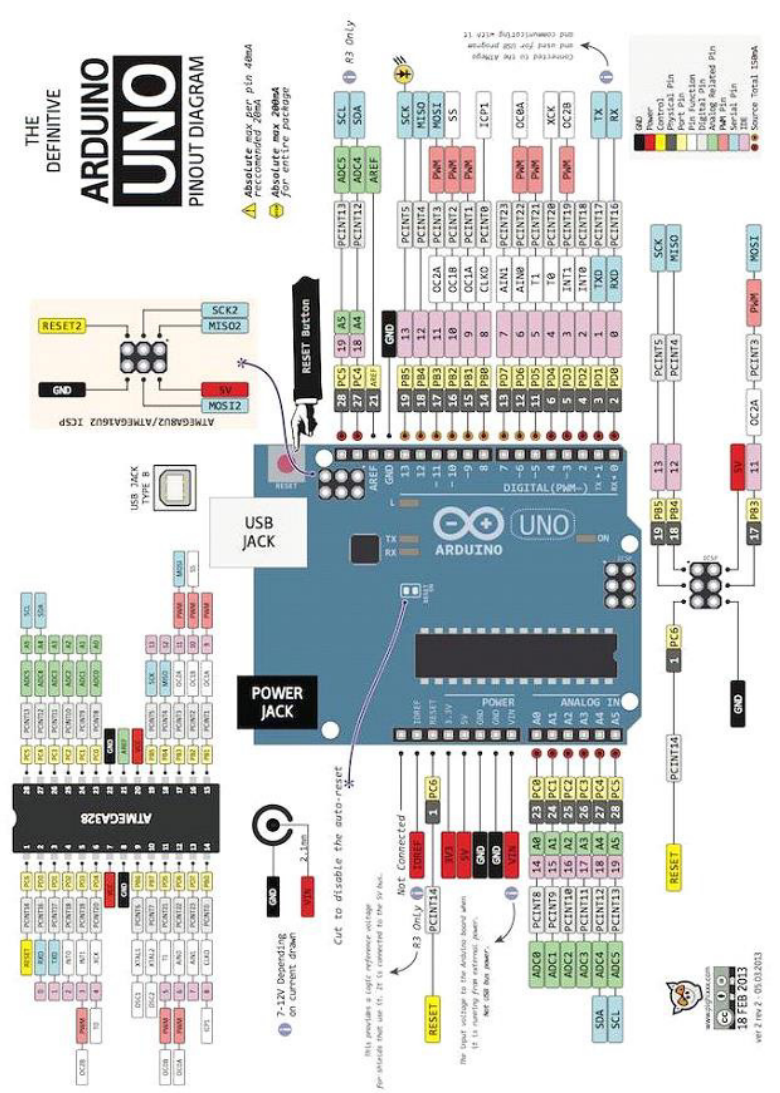

Fig. 3. ArduinoUNO electrical circuit diagram.

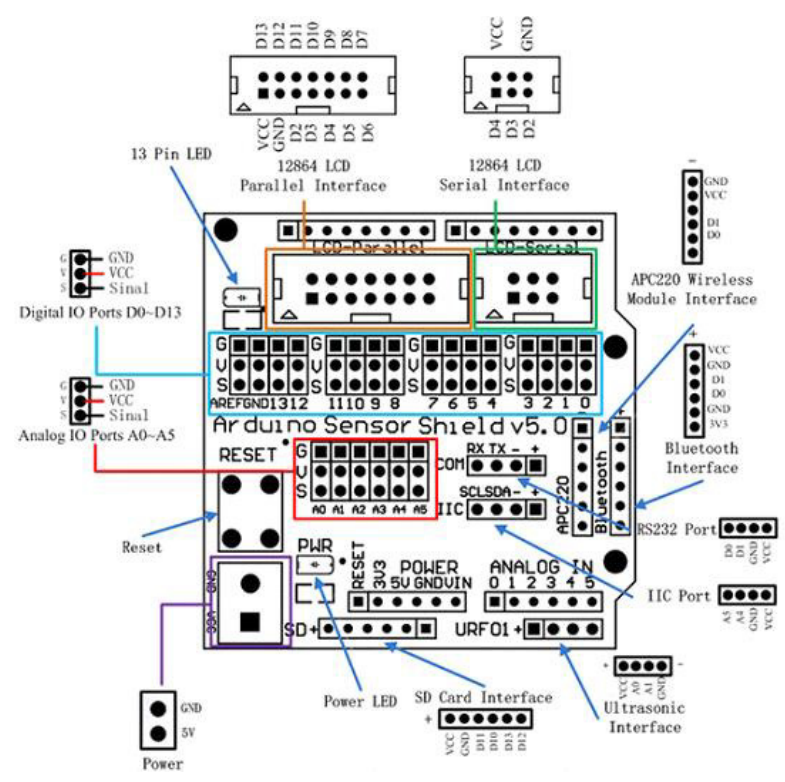

Fig. 4. Arduino sensor shield v5 functional diagram. 


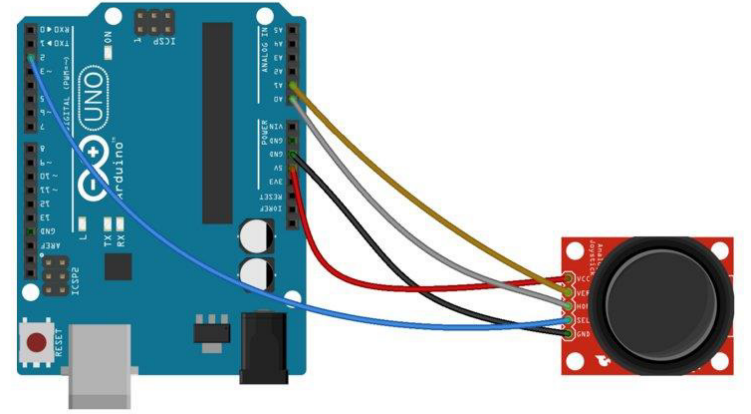

Fig. 5. PS2 stick module's functional diagram.

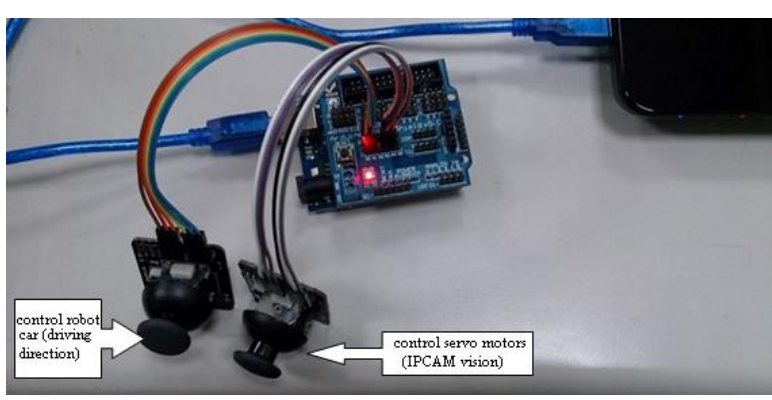

Fig. 6. Functions of the PS2 stick.

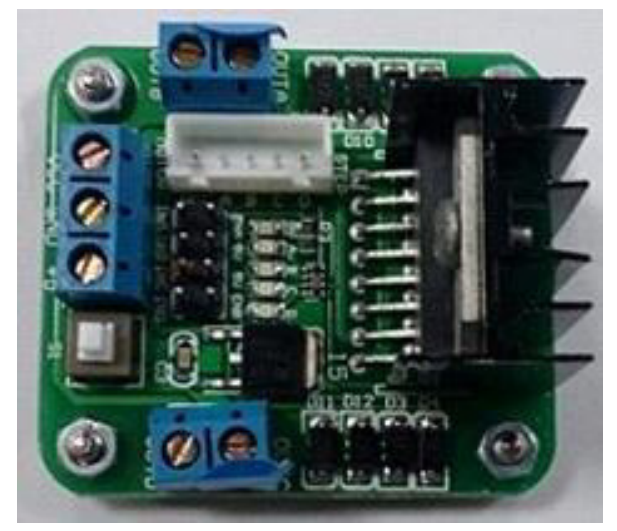

Fig. 7. Hardware of the motor driver board and the Arduino expanded board.

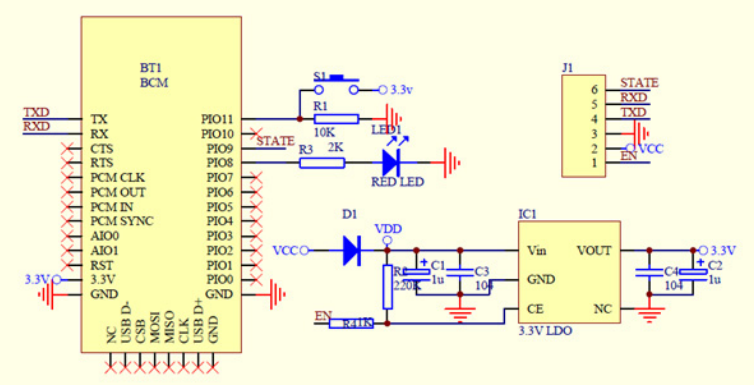

Fig. 8. Electrical circuit for the blue tooth protocol.
Table 1. The corresponding pins of the blue tooth with respect to the Arduino expanded board.

\begin{tabular}{|c|c|c|c|}
\hline $\begin{array}{c}\text { pin of blue } \\
\text { tooth }\end{array}$ & $\begin{array}{c}\text { pin of } \\
\text { Arduino } \\
\text { expanded } \\
\text { board }\end{array}$ & $\begin{array}{c}\text { pin of blue } \\
\text { tooth }\end{array}$ & $\begin{array}{c}\text { pin of Arduino } \\
\text { expanded } \\
\text { board }\end{array}$ \\
\hline VCC & COM(+) & TXD & COM(RX) \\
\hline GND & COM(-) & RXD & COM(TX) \\
\hline
\end{tabular}

Table 2. The corresponding pins of the motor driven board with respect to the Arduino expanded board.

\begin{tabular}{|c|c|c|c|}
\hline $\begin{array}{c}\text { pin of } \\
\text { motor } \\
\text { driven } \\
\text { board }\end{array}$ & $\begin{array}{c}\text { pin of motor } \\
\text { driven board } \\
\text { Arduino } \\
\text { expanded } \\
\text { board }\end{array}$ & $\begin{array}{c}\text { pin of motor } \\
\text { driven } \\
\text { board }\end{array}$ & $\begin{array}{c}\text { pin of motor } \\
\text { driven board } \\
\text { Arduino } \\
\text { expanded } \\
\text { board }\end{array}$ \\
\hline ENA & 6 & OUTA & $\begin{array}{c}\text { pole(left } \\
\text { side) }\end{array}$ \\
\hline INA & 4 & OUTB & $\begin{array}{c}\text { pole(left } \\
\text { side) }\end{array}$ \\
\hline INB & 5 & OUTC & $\begin{array}{c}\text { pole(right } \\
\text { side) }\end{array}$ \\
\hline INC & 7 & OUTD & $\begin{array}{c}\text { pole(right } \\
\text { side) }\end{array}$ \\
\hline IND & 8 & & \\
\hline ENB & 11 & & \\
\hline
\end{tabular}

Table 3. The corresponding pins of the PS2 stick board (for the carrier's motor control) with respect to the Arduino expanded board.

\begin{tabular}{|c|c|c|c|}
\hline $\begin{array}{c}\text { pin of PS2 } \\
\text { stick board } \\
\text { (for } \\
\text { carrier's } \\
\text { motor } \\
\text { control) }\end{array}$ & $\begin{array}{c}\text { pin of motor } \\
\text { driven board } \\
\text { Arduino } \\
\text { expanded } \\
\text { board }\end{array}$ & $\begin{array}{c}\text { pin of PS2 } \\
\text { stick board } \\
\text { (for carrier's } \\
\text { motor } \\
\text { control) }\end{array}$ & $\begin{array}{c}\text { pin of motor } \\
\text { driven board } \\
\text { Arduino } \\
\text { expanded } \\
\text { board }\end{array}$ \\
\hline S-Y & A1 & S-X & A0 \\
\hline S-Y -VCC & V & S-X -VCC & V \\
\hline S-Y -GND & G & S-X -GND & G \\
\hline S-K & N/A & S-K -GND & N/A \\
\hline S-K -VCC & N/A & & \\
\hline
\end{tabular}

Table 4. The corresponding pins of the PS2 stick board (for the IPCAM's servo motor control) with respect to the Arduino expanded board.

\begin{tabular}{|c|c|c|c|}
\hline $\begin{array}{c}\text { pin of PS2 } \\
\text { stick board } \\
\text { (for }\end{array}$ & $\begin{array}{c}\text { pin of motor } \\
\text { driven board } \\
\text { Arduino } \\
\text { IPCAM's } \\
\text { servo motor } \\
\text { bonted }\end{array}$ & $\begin{array}{c}\text { pin of PS2 } \\
\text { stick board } \\
\text { (for } \\
\text { IPCAM's } \\
\text { servo motor } \\
\text { control) }\end{array}$ & $\begin{array}{c}\text { pin of motor } \\
\text { driven board } \\
\text { Arduino } \\
\text { expanded } \\
\text { board }\end{array}$ \\
\hline S-Y & A4 & S-X & A3 \\
\hline S-Y -VCC & V & S-X -VCC & V \\
\hline S-Y -GND & G & S-X -GND & G \\
\hline S-K & N/A & & \\
\hline S-K -VCC & N/A & & \\
\hline S-K -GND & N/A & & \\
\hline
\end{tabular}

\section{Human/machine interface}

Using the RS-232 communication standard and blue tooth protocol, the server port (server pc) is wirelessly connected to the microcontroller (single chip). Here, microcontroller connects to the actuators (servo motors, DC motors) and the toxic gas sensor (methane detector). 
As indicated in Figure 2, the IPCAM connected to the wireless AP router will capture the image and send it back to the PC server. As shown in Figure 1, the PS2 module used to control both the carrier's direction and the IPCAM's vision is linked to the server PC via the USB protocol.

To remotely manipulate the robot car and implement visual monitoring/gas detecting, an interface of the server PC programmed by Microsoft VB2010 is required. As illustrated in Figure 9, the interface of the server PC is initiated. The control panel (at the left and lower side) for manipulating the direction of the carriern will be enabled when the "COM No." is inputted and the "connect" button is clicked. Subsequently, the concentration of the toxic gas (methane) detected by the gas sensor will be sent back and shown on the server PC's interface (at the left and lower side). Alternatively, as depicted in Figure 10, the PS2's stick will replace the server PC's manipulation when the PC's control connection is terminated, the PS2's COM number is inputted, and the PS2's connect button is clicked. At this time, the user can freely manipulate the carrier and the IPCAM's rotation by using the PS2 stick. As indicated in Figure 11, the system will be terminated when clicking the "exit" button.

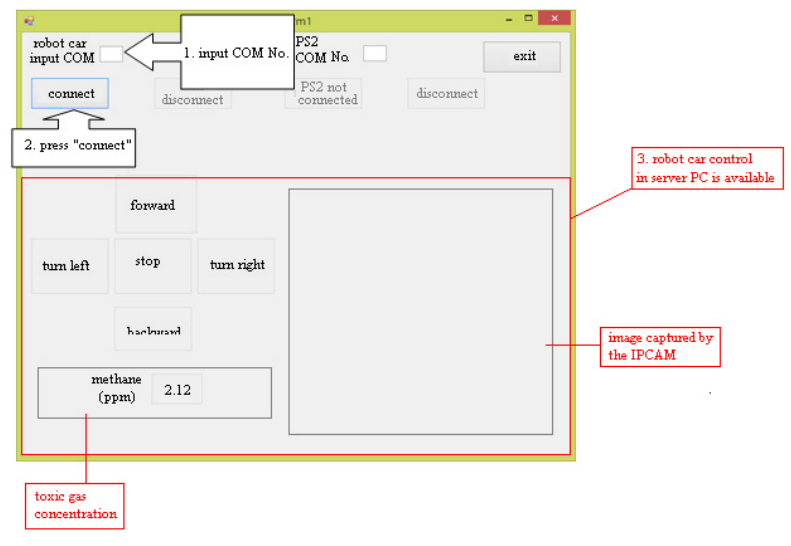

Fig. 9. Interface in server PC port.

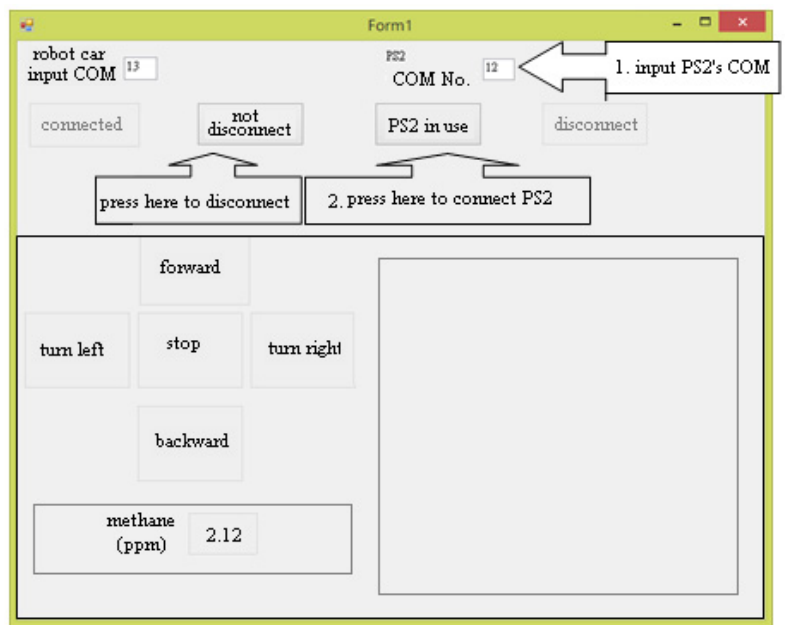

Fig. 10. Interface for connecting to the PS2 port.

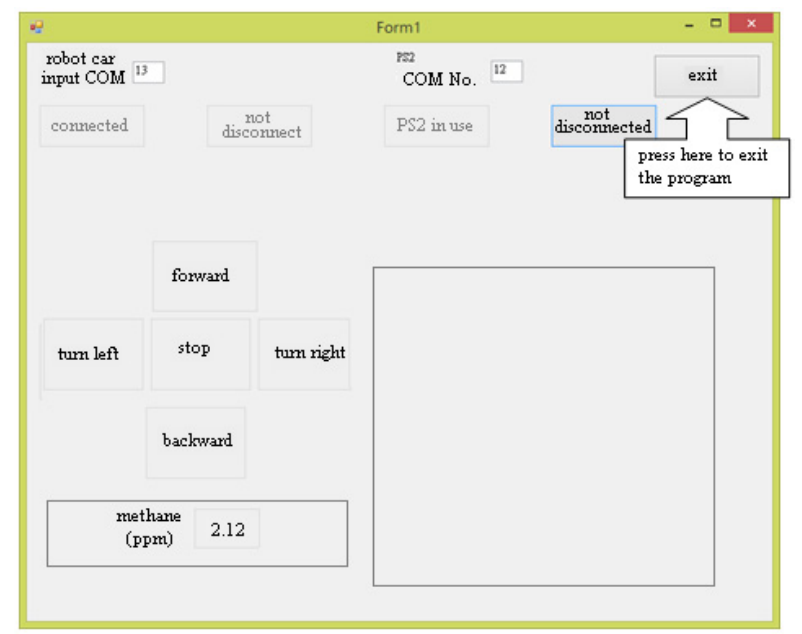

Fig. 11. Interface for exit.

\section{Results and Discussion}

A robot car system equipped with a server PC control port, a stick control port, a visual port, and a robot car has been demonstrated. The patrolling path can be remotely controlled via the server PC control port and stick control port. The IPCAM's vision can be adjusted by controlling the servo motors via the server PC control port and stick control port. Both toxic gas concentration and environmental vision can be obtained and fedback to the server PC. As indicated in Figure 12(a)-(e), the toxic gas concentration has been detected and sent back to the server PC's interface. The environmental vision has been captured by the IPCAM and fedback to the interface. The user can warn the plant via a broadcasting device when either the toxic gas emissions or security problems arrive.

The site testing for the wireless path-patrol and toxic gas detecting security robot car is shown in Figure 13.

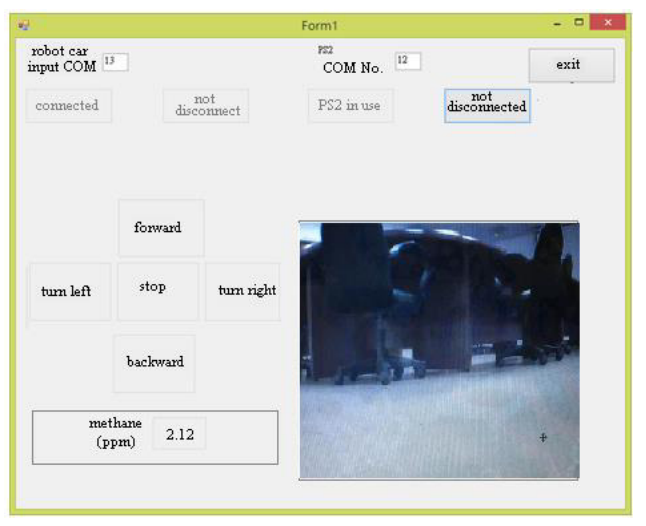

Fig. 12(a). Remote toxic gas detecting and environmental security monitoring - Vision capture\#1. 


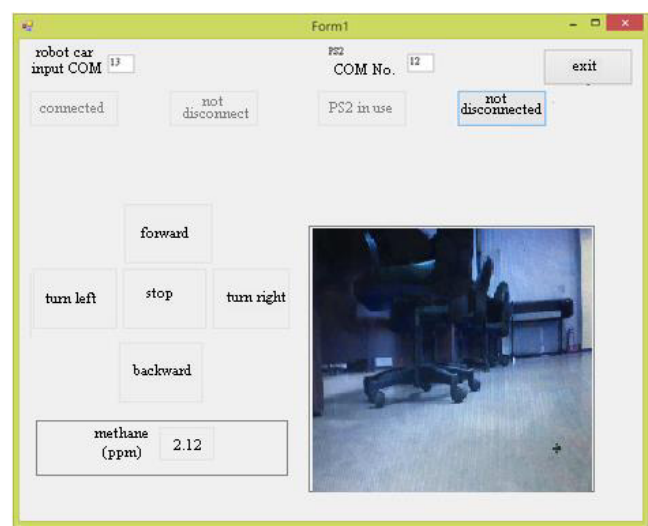

Fig. 12(b). Remote toxic gas detecting and environmental security monitoring - Vision capture $\# 2$.

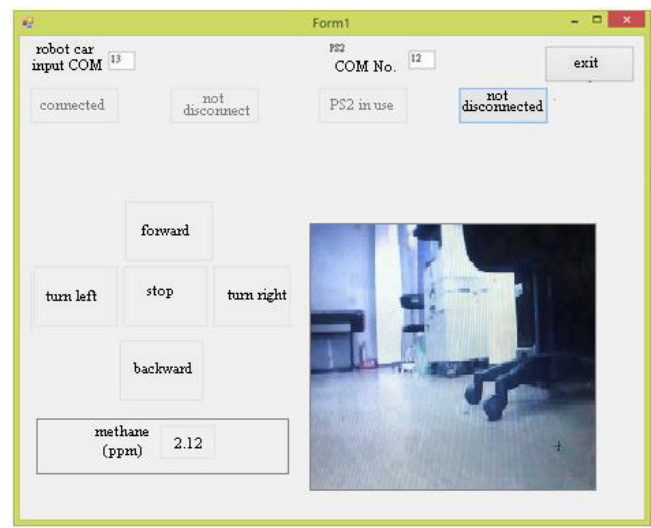

Fig. 12(c). Remote toxic gas detecting and environmental security monitoring - Vision capture\#3.

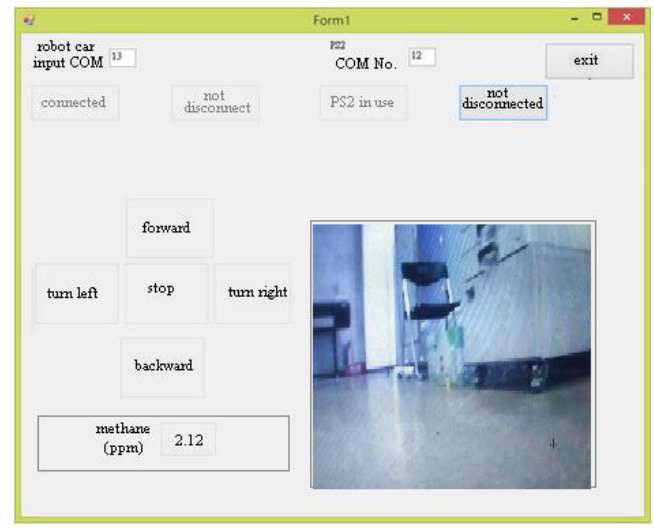

Fig. 12(d). Remote toxic gas detecting and environmental security monitoring - Vision capture\#4.

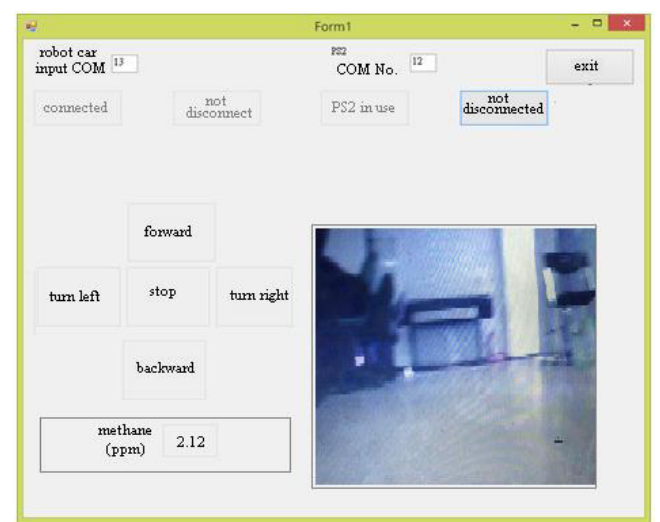

Fig. 12(e). Remote toxic gas detecting and environmental security monitoring - Vision capture $\# 5$.

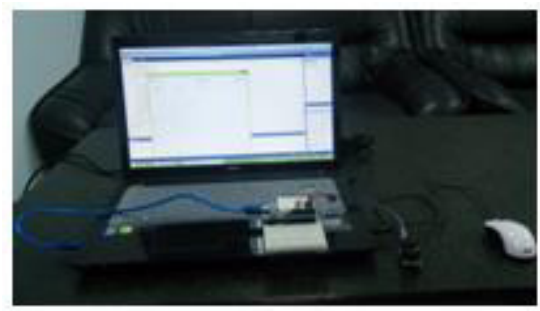

Fig. 13(a). Operational result of a wireless Path-Patrol and Toxic Gas Detecting Security robot car. - server PC port.

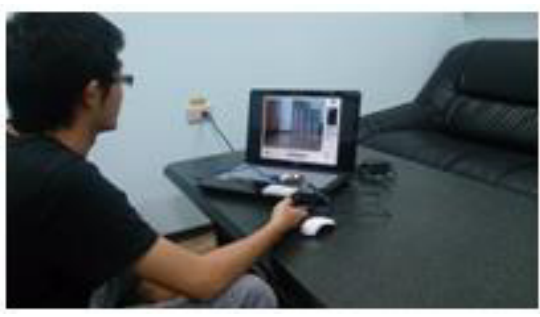

Fig. 13(b). Operational result of a wireless Path-Patrol and Toxic Gas Detecting Security robot car. - manipulating in server PC.

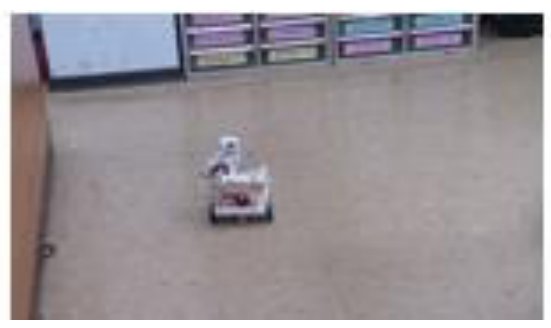

Fig. 13(c). Operational result of a wireless Path-Patrol and Toxic Gas Detecting Security robot car. - robot car moving.

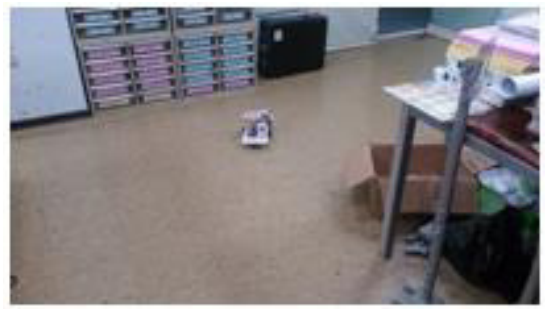

Fig. 13(d). Operational result of a wireless Path-Patrol and Toxic Gas Detecting Security robot car. - robot car moving. 


\section{Conclusion}

It has been shown that a wireless path-patrol and toxic gas detecting security robot car can remotely monitor vision and toxic gas emissions in a plant. Using the RS232 communication standard and blue tooth protocol, the server port (server pc) is wirelessly connected to the microcontroller (single chip). The microcontroller connects to the gas sensor (methane sensor) and four outputs (two servo motors and two DC motor). In addition, in order to remotely monitor environmental status, a wireless USB AP router wirelessly connected to the IPCAM is adopted. The image can be sent back to the server PC via the Wi-Fi protocol. To implement the path-patrol and toxic gas detecting security work, a human/machine interface in the server PC port programmed by Microsoft VB 2010 has been established and shown in Figures 9-11. A user can manipulate the robot car's patrolling path and adjust the IPCAM's vision angle via the server PC port or the PS2 stick port. Additionally, the current image shown in Figure 12 can also be captured and sent back by the IPCAM via the Wi-Fi protocol. Moreover, a user can remotely manipulate the robot car to detect gas emissions without danger. A warning via the broadcast device from the control center will be sent to the whole plant when either toxic gas emissions or security problem occur.

Consequently, a prototype of the wireless path-patrol and toxic gas detecting security robot car to ensure workers' safety and plant security is established.

\section{References}

1. I. O. Osunmakinde, Towards safety from toxic gases in underground mines using wireless sensor networks and ambient intelligence, I. J. of Distributed Sensor Networks, Article ID 159273, 115, (2013)

2. K. Steenland, Diesel exhaust and lung cancer in the trucking industry: Exposure-response analysis and risk assessment, Am. J. Ind. Med., 34, 220-228, (1998)

3. S. Lind, Types and sources of fatal and severe nonfatal accidents in industrial maintenance, I. J. of Industrial Ergonomics, 38, 927-933, (2008)

4. A. J. Cohen, H. R. Anderson, B. Ostro, K. D. Pandey, M. Krzyzanowski, N. Kunzli, K. Gutschmidt, A. Pope, I. Romieeu, J. M. Samet, K. Smith, The global burden of disease due to outdoor air pollution, J. of Toxicology and Environmental Health, Part A, 68, 1301-1307, (2005)

5. C. A. Pope, Lung Cancer, Cardiopulmonary mortality, and long-term exposure to fine particulate air pollution, $J$. of the American Medical Association, 287 (9), 1132-1141, (2002)

6. C. A. Pope, Epidemiology of fine particulate air pollution and human health: Biologic mechanisms and who's at risk?, Environmental Health Perspectives, 108, 713-723, (2000)
7. D. Pokhsraryan, V. Aroutiounian, H. Chilingaryan, Gas monitoring system, Armenian J. of Physics, 3(1), 78-81, (2010)

8. H. Navale, Arm based gas monitoring system, I. J. of Scientific and Technology Research, 3(6), 43-45, (2014)

9. M. Xiaomin, Recognition of toxic gases emission in power plant based on artificial neural network, Energy Procedia, 17, 1578-1584, (2012)

10. M. C. Chiu, L. J. Yeh, Y. C. Lin, The design and application of a robotic vacuum cleaner, J. of Information \& Optimization Sciences, 30(1), 39-62, (2009)

11. M. C. Chiu, Numerical assessment of path-planning for an autonomous robot passing through multi-layer barrier systems using a genetic algorithm, Information Technology J., 9(7), 1483-1489, (2010)

12. M. C. Chiu, Path planning for an autonomous robot using a simulating annealing, J. of Information \& Optimization Sciences, 32(2), 297-314, (2011)

13. M.C. Chiu, Numerical Assessment of path planning for autonomous robotic vacuum cleaner using an ant colony optimization method, J. of Information and Optimization Science, 32(5), 1039-1058, (2011)

14. M. C. Chiu, J. H. Wen, Wall plug-in power supply system for a robotic vacuum cleaner, $J$. of Information \& Optimization Sciences, 32(2), 2772951, (2011)

15. M. C. Chiu, L. J. Yeh, C. C. Shih, Automatic Cable Spooling for a Robotic Vacuum Cleaner, J. of Information \& Optimization Sciences, 31(3), 543$559,(2010)$

16. M. C. Chiu, L. J. Yeh, T. S. Lan, W. C. Liao, C. H. Chung, Ground-purity inspection for a grouped robotic cleaner, The Canadian Society for Mechanical Engineering (CSME) Transactions, 36(2), 161-172, (2012)

17. M. C. Chiu, L.J. Yeh, T.S. Lan, S.C. Yen, Positioning and path planning of a swarm robotic cleaner, Advanced Materials Research, 740, 112119, (2013)

18. T. S. Lan, L. J. Yeh, M. C. Chiu, Y. X. Hwang, Construction of the control system of cleaning robots with vision guidance, Mathematical Problems in Engineering, 283(795), 1-6, (2013)

19. M. C. Chiu, L. J. Yeh, G. J. Lai, B. M. Huang, Developing an auto-tracking carrier of AGV using a wave-varied detecting method, Proc. ImechE Part C: J. of Mechanical Engineering Science, 224(C6), 1349-1357, (2010)

20. M. C. Chiu, T. S. Lan, S. F. Hwang, Development of a real-time task editable service-oriented security robot, J. of Interdisciplinary Mathematics, 15(4\&5), 239-259, (2012) 